\title{
Determination of static fracture toughness of coarse-grained and ultrafine-grained materials by the depth of the plastic zone under the fractures surface
}

\author{
G. V. Klevtsov ${ }^{\dagger, 1}$, R. Z. Valiev' ${ }^{2}$, N. A. Klevtsova ${ }^{1}$, I. N. Pigaleva ${ }^{1}$ \\ ${ }^{\dagger}$ Klevtsov11948@mail.ru
}

${ }^{1}$ Togliatti State University, Togliatti, 445667, Russia

${ }^{2}$ Research Institute of Advanced Materials Physics, Ufa State Aviation Technical University, Ufa, 455000, Russia

\begin{abstract}
In the practice of diagnostics of emergency failure of structures or machine parts, there are cases when information is needed on the fracture toughness $\left(K_{1 \mathrm{C}}\right.$ or $\left.K_{\mathrm{C}}\right)$ of the material of products, the dimensions and configuration of which do not allow the fabrication of samples for testing the material for $K_{1 C}$ according to the standard GOST 25.506-85. On an example of a large group of materials with bcc, fcc and hcp lattices, the relationship of the fracture toughness $\left(K_{1 \mathrm{C}}\right.$ or $\left.K_{\mathrm{C}}\right)$ of materials with the yield strength, hardness and elongation is considered. It is shown that the fracture toughness of materials both in coarsegrained (CG) and ultrafine-grained (UFG) states is ambiguously related to these mechanical characteristics of materials. Therefore, the fracture toughness of materials cannot be determined by calculation based on the above strength and plastic properties. In this paper, we propose a method for determining fracture toughness of CG and UFG materials by the maximum depth of the plastic zone under the fracture surface $\left(h_{\max }\right)$ of fractured specimens, structures, or machine parts. The depth of the plastic zones under the fracture surface was determined by the X-ray method. If the destruction occurred in the plane stress (PS) condition or in the transition region from plane deformation to plane stress state (PD $\leftrightarrow \mathrm{PS}$ ), then the depth of the slightly deformed macro zone $\left(h_{\mathrm{y}}\right)$ was taken as $h_{\max }$. Using the example of a large group of materials with bcc, fcc and hcp lattices, it has been shown that it's possible in principle to determine the fracture toughness of CG and UFG materials with different types of crystal lattice using the plastic zone depth under the fractures surface.
\end{abstract}

Keywords: fracture toughness $\left(K_{1 \mathrm{C}}\right.$ or $\left.K_{\mathrm{C}}\right)$, local stress state, plastic zone depth, UFG and CG materials.

УДК: 621.7(07)

\section{Определение статической трещиностойкости крупнозернистых и ультрамелкозернистых материалов по глубине пластической зоны под поверхностью изломов}

\author{
Клевцов Г. В. ${ }^{\dagger, 1}$, Валиев Р. 3. ${ }^{2}$, Клевцова Н. А. ${ }^{1}$, Пигалева И. Н. \\ ${ }^{1}$ Тольяттинский государственный университет, Тольятти, 445667, Россия \\ ${ }^{2}$ НИИ Физики перспективных материалов, Уфимский государственный авиационный технический университет, \\ Уфа, 455000, Россия
}

В практике диагностики аварийного разрушения конструкций или деталей машин бывают случаи, когда необходимы сведения о трещиностойкости $\left(K_{1 \mathrm{C}}\right.$ или $\left.K_{\mathrm{C}}\right)$ материала изделия, размеры и конфигурация которого не позволяют изготовить образцы для испытания материала на $K_{1 \mathrm{C}}$ по ГОСТ 25.506-85. На примере большой группы материалов с ОЦК, ГЦК и ГПУ решеткой рассмотрена связь статической трещиностойкости $\left(K_{1 \mathrm{C}}\right.$ или $\left.K_{\mathrm{C}}\right)$ материалов с пределом текучести, твердостью и относительным удлинением. Показано, что статическая трещиностойкость материалов как в крупнозернистом (КЗ) состоянии, так и в ультрамелкозернистом (УМЗ) состоянии неоднозначно связана с данными механическими характеристиками материалов. Следовательно, статическая трещиностойкость материалов не может быть определена путем расчета, основанного на вышеуказанных прочностных и пластических свойствах. В настоящей работе предложена методика оценки статической трещиностойкости К3 и УМЗ материалов по максимальной глубине пластической зоны под поверхностью изломов $\left(h_{\max }\right)$ разрушенных образцов, 
конструкций или деталей машин. Для оценки статической трещиностойкости материалов по глубине пластической зоны предложено использовать уравнение, где коэффициент $n$ характеризует локальное напряженное состояние материала у вершины трещины. Глубину пластических зон под поверхностью изломов определяли рентгеновским методом. Если разрушение произошло в условиях плоского напряженного состояния (ПН) или в переходной области от плоской деформации к плоскому напряженному состоянию (ПДњПН), то за $h_{\max }$ принимали глубину слабодеформированной макрозоны $\left(h_{\mathrm{y}}\right)$. На примере большой группы материалов с ОЦК, ГЦК и ГПУ решеткой показана принципиальная возможность оценки статической трещиностойкости КЗ и УМЗ материалов с различным типом кристаллической решетки по глубине пластической зоны под поверхностью изломов.

Ключевые слова: статическая трещиностойкость $\left(K_{1 \mathrm{C}}\right.$ или $\left.K_{\mathrm{C}}\right)$, локальное напряженное состояние, глубина пластической зоны, УМЗ и КЗ материалы.

\section{1. Введение}

Определение статической трещиностойкости материалов $\left(K_{1 \mathrm{C}}\right.$ или $\left.K_{\mathrm{C}}\right)$ с оценкой локального напряженного состояния у вершины трещины, в том числе и материалов с ультрамелкозернистой (УМЗ) структурой [1-7], имеет принципиальное значение, например, при диагностике причин аварийного разрушения конструкций и деталей машин. Однако в практике диагностики аварийного разрушения бывают случаи [8-11], когда необходимы сведения о трещиностойкости материала изделия, размеры и конфигурация которого не позволяют изготовить образцы для испытания материала на $K_{1 \mathrm{C}}$ по ГОСТ 25.506-85. В этом случае необходимы альтернативные методы оценки трещиностойкости материала: либо путем пересчета, исходя из таких характеристик материала как предел прочности или текучести, либо необходимы принципиально иные методики оценки трещиностойкости материалов разрушенных образцов, конструкций или деталей машин.

Согласно положению механики разрушения, существует корреляционная связь трещиностойкости материала и локального напряженного состояния материала в момент разрушения с размером и формой пластических зон у вершины трещины $[12,13]$. Различают два предельных значения локального напряженного состояния материала: плоскую деформацию (ПД) и плоское напряженное состояние (ПН). Однако на практике разрушение металлических материалов нередко происходит в переходной области (ПДњПН) [9,14]. Принимая во внимание важность такого параметра как размер пластической зоны у вершины трещины, в работах $[9,14-16]$ предложен критерий оценки локального напряженного состояния материала как отношение максимальной глубины пластической зоны под поверхностью изломов $h_{\max }$ к толщине образца или детали $t$, т.е. $h_{\max } / t$. Показано $[9,14-16]$, что разрушение материалов в условиях ПД сопровождается образованием у вершины трещины одной пластической зоны $\left(h_{\mathrm{y}}\right)$; при этом $h_{\max } / t<10^{-2}$. В условиях ПН у вершины трещины образуются две пластические зоны: слабодеформированная макрозона $\left(h_{\mathrm{y}}\right)$ и сильнодеформированная микрозона $\left(h_{\mathrm{yh}}\right)$; при этом $h_{\max } / t>10^{-1}$. $\mathrm{B}$ переходной области (ПД $\leftrightarrow$ ПН) под поверхностью изломов также выявляются две пластические зоны, однако не всегда представляется возможным разделить макро- и микрозону [9]. В данной области выполняется условие $10^{-2} \leq h_{\max } / t \leq 10^{-1}$.
Целью настоящей работы является разработка методики оценки статической трещиностойкости материалов по глубине пластической зоны под поверхностью изломов разрушенных образцов, конструкций или деталей машин.

\section{2. Материалы и методики исследования}

В настоящей работе проанализированы ранее представленные в работах $[6,9,15,17-19]$ значения статической трещиностойкости материалов и глубины пластической зоны под поверхностью изломов для КЗ материалов с размером зерен от 10 до 60 мкм и УМЗ материалов, полученных путем интенсивной пластический деформации (ИПД), с размером зерен от 0.3 до 0.9 мкм и высокой плотностью дефектов кристаллического строения.

Испытание всех сталей и сплавов на статическую трещиностойкость проводили согласно ГОСТ 25.506-85 либо на призматических образцахпо схеме трехточечного изгиба, либо на образцах на внецентренное растяжение. Глубину пластических зон под поверхности изломов определяли рентгеновским методом $[9,16]$. Если разрушение произошло в условиях ПН или в переходной области (ПД $\leftrightarrow$ ), то за $h_{\max }$ принимали глубину слабодеформированной макрозоны $\left(h_{\mathrm{y}}\right)$ [9].

\section{3. Результаты исследования и их обсуждение}

Рассмотрим на примере большой группы материалов с ОЦК, ГЦК и ГПУ решеткой связь статической трещиностойкости $\left(K_{1 \mathrm{C}}\right.$ или $\left.K_{\mathrm{C}}\right)$ с пределом текучести, твердостью и относительным удлинением (Рис. $1 \mathrm{a}-\mathrm{c}$ ). Из Рис. 1 видно, что статическая трещиностойкость материалов как в КЗ, так и в УМЗ состоянии неоднозначно связана с данными механическими характеристиками материалов. Следовательно, статическая трещиностойкость материалов не может быть определена путем расчета, основанного на вышеуказанных прочностных и пластических свойствах. Обращает на себя внимание, что условие ПД по критерию $t /\left(K_{1 \mathrm{C}} / \sigma_{0.2}\right)^{2} \geq 2.5$ выполняется в средней области прочностных и пластических характеристик представленных материалов (Рис. 1a-c).

Известно, что в механике разрушения связь радиуса пластической зоны у вершины трещины с коэффициентом интенсивности напряжения выражена уравнениями: для ПН $r=1 / 2 \pi \cdot\left(K_{\mathrm{C}} / \sigma_{0.2}\right)^{2}$; для ПД 
$r_{1}=1 / 6 \pi \cdot\left(K_{1 \mathrm{C}} / \sigma_{0.2}\right)^{2}[12,13]$. В работе [20] на основании исследования широкого класса КЗ материалов с ОЦК и ГЦК структурой нами было предложено оценивать статическую трещиностойкость К3 материалов ( $K_{1 \mathrm{c}}$ или $K_{\mathrm{C}}$ ) по глубине пластической зоны под поверхностью изломов $\left(h_{\max }\right)$, используя уравнение:

$$
h_{\max }=\frac{1}{n \pi}\left(\frac{K_{1 \mathrm{C}}\left(K_{\mathrm{C}}\right)}{\sigma_{0.2}}\right)^{2},
$$

где коэффициент $n$ характеризует локальное напряженное состояние материала у вершины трещины по критерию $h_{\max } / t$.

Построим зависимость « $h_{\max } / t-n »$ (Рис. 2), используя значения $\left(K_{1 \mathrm{C}}\right.$ или $\left.K_{\mathrm{C}}\right)$, полученные в процессе испытания на статическую трещиностойкость КЗ и УМЗ материалов с различным типом кристаллической решетки и значения максимальной глубины пластической зоны под поверхностью изломов $\left(h_{\max }\right)$.

В условиях ПН $\left(h_{\max } / t>10^{-1}\right)$ (Рис. 2) все рассматриваемые материалы разрушаются вязко с образованием ямочного микрорельефа. В этом случае, независимо от состояния материалов (КЗ или УМЗ), коэффициент $n=2$ и совпадает с расчетным значением механики разрушения $[12,13]$. В переходной области ПНњПД $\left(10^{-2} \leq h_{\max } / t \leq 10^{-1}\right)$ (Рис. 2) материалы с ОЦК решеткой разрушаются крайне редко [9], а материалы с ГЦК и ГПУ решеткой при одинаковом уровне локального напряженного состояния имеют одинаковое значение коэффициента $n$. Из-за высокой пластичности добиться разрушения материалов с ГЦК решеткой в условиях ПД не представилось возможным. Значения коэффициента $n$ для материалов с ОЦК и ГПУ решеткой в условиях
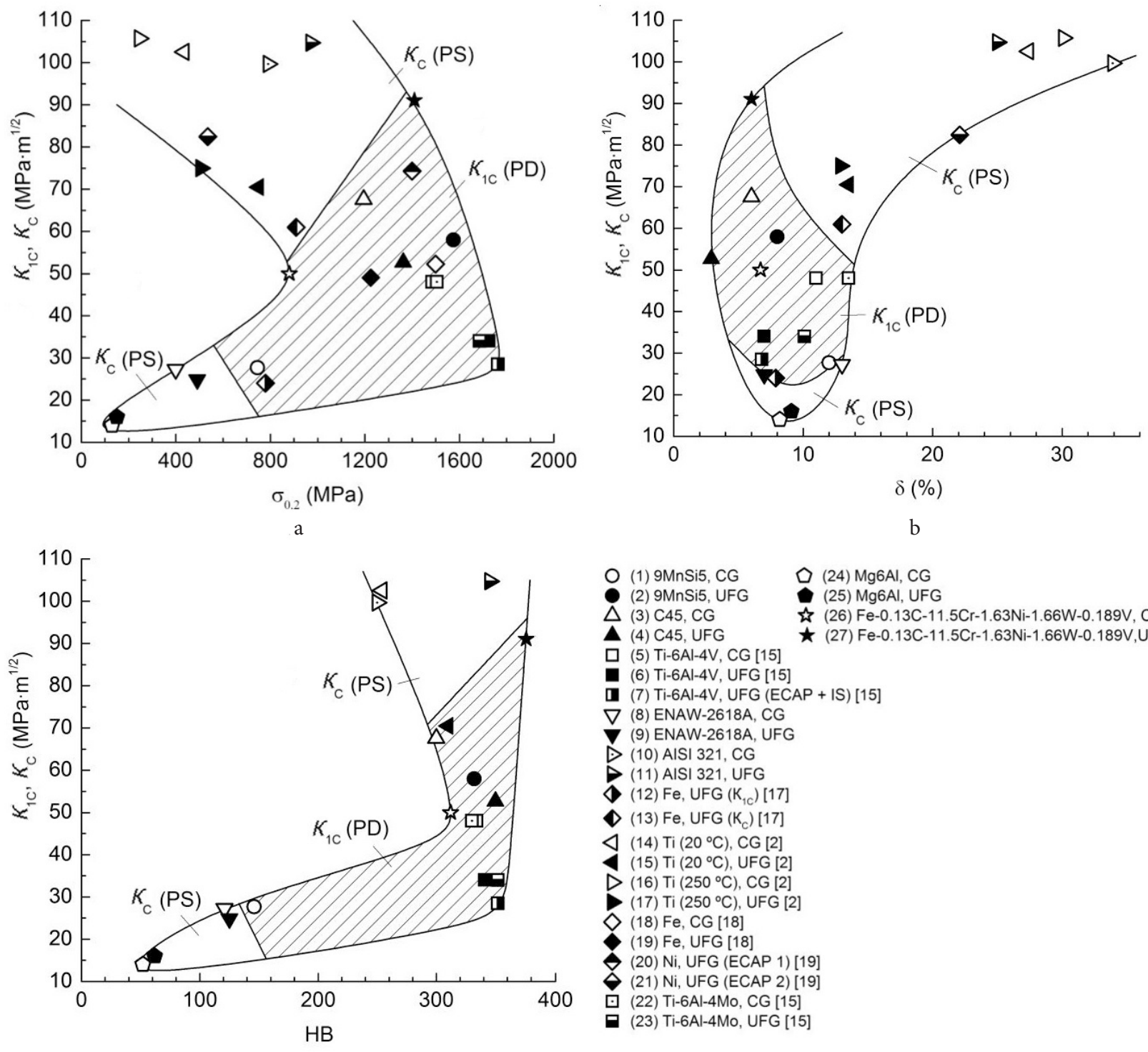

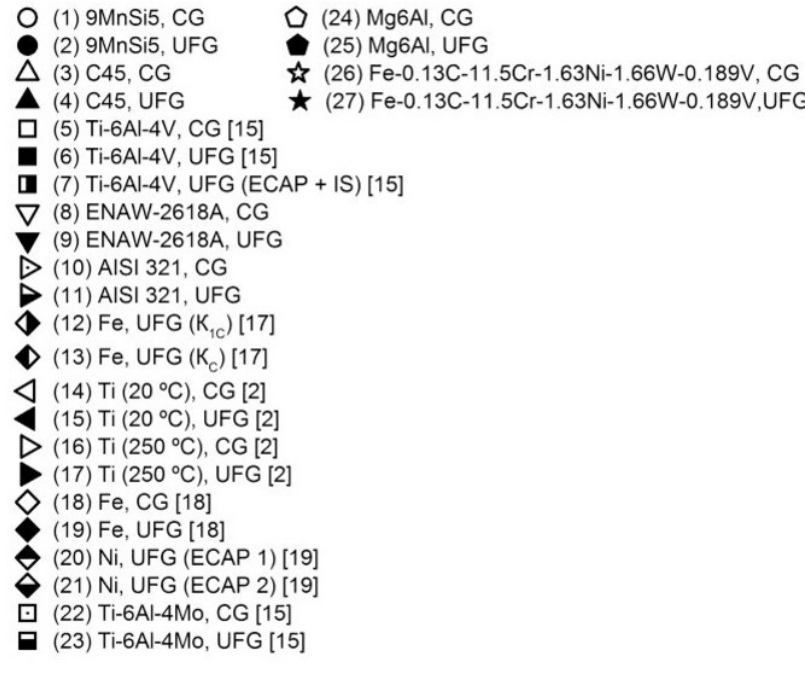

Pис. 1. Связь статической трещиностойкости $\left(K_{1 \mathrm{C}}\right.$ и $\left.K_{\mathrm{C}}\right)$ с пределом текучести (a), относительным удлинением (b) и твердостью (c) КЗ и УМЗ материалов с ОЦК $(1-4,12,13,18,19,26,27)$, ГЦК $(8-11,20,21)$ и ГПУ $(5-7,14-17,22-25)$ решеткой. Заштрихованная область соответствует условию ПД по критерию $t /\left(K_{1 \mathrm{C}} / \sigma_{0.2}\right)^{2} \geq 2.5$.

Fig. 1. The relationship of fracture toughness $\left(K_{1 C}\right.$ and $\left.K_{C}\right)$ with yield strength (a), relative elongation (b) and hardness (c) of CG and UFG materials with bcc $(1-4,12,13,18,19,26,27)$, fcc $(8-11,20,21)$ and hcp $(5-7,14-17,22-25)$ lattice. The hatched region corresponds to the PD condition by the criterion $t /\left(K_{1 \mathrm{C}} / \sigma_{0.2}\right)^{2} \geq 2.5$. 
ПД $\left(h_{\max } / t<10^{-2}\right)$ (Рис. 2) существенно различаются. Это связано, по-видимому, с наличием вязко-хрупкого перехода в материалах с ОЦК решеткой и отсутствием такового в материалах с ГПУ решеткой [9,21]. Так, например, сталь 09MnSi5 (материал с ОЦК решеткой) в условиях ПД разрушается хрупко по механизму скола в КЗ состоянии (Рис. 3 а) или квазискола в УМЗ состоянии (Рис. 3 b). Титановый сплав Ti-6Al-4Mo (материал с ГПУ решеткой) в условиях ПД не охрупчивается независимо от состояния. Сплав разрушается с образованием хаотично расположенных сравнительно гладких гребней (Рис. 3 с, d) [6,15].

Уравнение (1) и представленные экспериментальные данные дают принципиальную возможность решить практическую задачу: определение статической трещиностойкости $\left(K_{1 \mathrm{C}}\right.$ или $\left.K_{\mathrm{C}}\right)$ КЗ и УМЗ материала разрушенного объекта с различным типом кристаллической решетки по глубине пластической зоны под поверхностью изломов. Для этого рентгеновским или иным методом следует определить максимальную глубину пластической зоны под поверхностью изломов $\left(h_{\max }\right)$ вблизи очага разрушения и замерить толщину образца, конструкции или детали в месте разрушения $(t)$. Затем рассчитать отношение $h_{\max } / t$ и по графику (Рис. 2) определить коэффициент $n$. Далее, используя уравнение (1), рассчитать $K_{1 \mathrm{C}}$ или $K_{\mathrm{C}}$ материала. Если $h_{\max } / t<10^{-2}$, то разрушение произошло в условиях ПД, a рассчитанное значение коэффициента интенсивности напряжения - есть величина $K_{1 \mathrm{c}}$.

Сравним на примере алюминиевого сплава АК4-1 в КЗ и УМЗ состояниях значение статической трещиностойкости, полученное при испытании сплава по ГОСТ 25.506-85 $\left(K_{\mathrm{C}}\right)$ и оцененное по предложенной методике $\left(K_{\mathrm{C}}^{\prime}\right)$ (Табл. 1). Сплав в К3 состоянии использовали после закалки и старения (Т6); УМЗ состоянии - после РКУП.

Из приведенной таблицы видно, что значение $K_{\mathrm{C}}^{\prime}$ сплава, оцененное по предложенной методики, близко к значению $K_{\mathrm{C}}$, полученному при испытании сплава по ГОСТ 25.506-85.

\section{4. Заключение}

Установлена связь коэффициента $n$, характеризующего локальное напряженное состояние материала у вер-

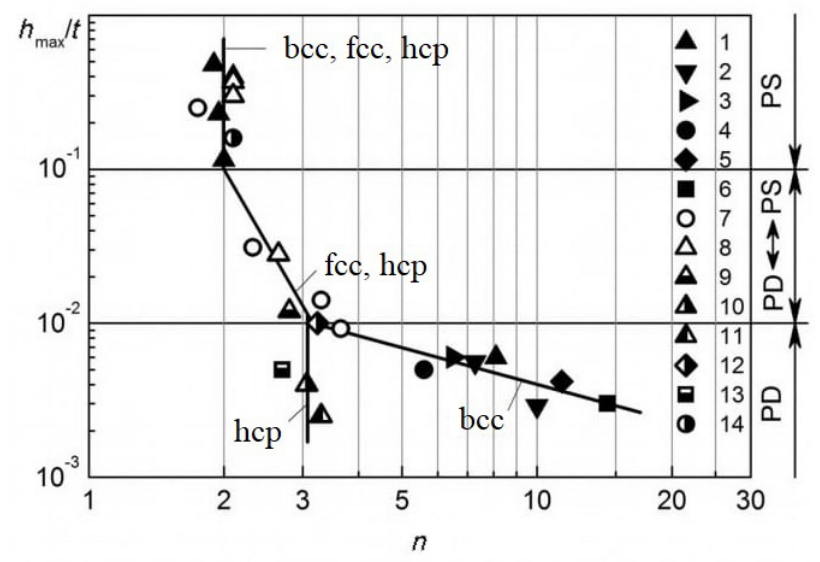

Рис. 2. Связь коэффициента $n$ с критерием $h / t$ для КЗ и УМЗ материалов с ОЦК $(1-6)$, ГЦК $(7,8)$ и ГПУ $(9-14)$ решеткой при испытании образцов на статическую трещиностойкость. К3 материалы: 1 - C20; 2 - C40; $4-$ Fe-0.15C-2Cr-1Mo-1V; 5 - 09MnSi5; 7 - ENAW-2024; 8 - Fe-0.03C-13Cr-0.1N-19Mn; 9 - Ti-6Al-4V; 12 - Ti-6Al-4Mo. УМ3 материалы: 3 - C45 (РКУП); 6 - 09MnSi5 (РКУП); $10-$ Ti-6Al-4V (РКУП); $11-$ Ti-6Al-4V (РКУП + ИЗТО); 13 - Ti-6Al-4Mо (ротационная ковка, РК); $14-\operatorname{Mg} 6 \mathrm{Al}$ (РКУП).

Fig. 2. The relationship of the coefficient $n$ with the criterion $h_{\max } / t$ for CG and UFG materials with bcc $(1-6)$, fcc $(7,8$.) and hcp $(9-14)$ lattice when testing samples for static crack resistance. CG materials: 1 - C20; 2 - C40; 4 - Fe-0.15C-2Cr-1Mo-1V; 5 - 09MnSi5; 7 ENAW-2024; 8 - Fe-0.03C-13Cr-0.1N-19Mn; 9 - Ti-6Al-4V; 12 - Ti-6Al-4Mo. UFG materials: 3 - C45 (ECAP); 6 - 09MnSi5 (ECAP); 10 - Ti-6Al-4V (ECAP); 11 - Ti-6Al-4V (ECAP + IZTO); 13 - Ti-6Al-4Mo (RC); $14-\operatorname{Mg} 6 \mathrm{Al}$ (ECAP).

Табл. 1. Сравнение значения $K_{\mathrm{C}}$ алюминиевого сплава АК4-1 [6], полученное при испытании сплава по ГОСТ 25.506-85 (K и оцененное по предложенной методике $\left(K_{\mathrm{C}}^{\prime}\right)$.

Table 1. Comparison of the $K_{\mathrm{C}}$ value of the AK4-1 aluminum alloy [6] obtained when testing the alloy according to GOST 25.506-85 $\left(K_{\mathrm{C}}\right)$ and estimated by the proposed method $\left(K_{\mathrm{C}}{ }^{\prime}\right)$.

\begin{tabular}{|c|c|c|c|c|c|}
\hline $\begin{array}{c}\text { Состояние } \\
\text { State }\end{array}$ & $\begin{array}{c}\sigma_{0.2}, \\
\mathrm{MPa}\end{array}$ & $\begin{array}{c}K_{\mathrm{C}}, \mathrm{M \Pi a} \sqrt{\mathrm{M}}_{\mathrm{M}}, \mathrm{MPa} \sqrt{\mathrm{m}} \\
K_{\mathrm{C}}\end{array}$ & $h_{\max } / t$ & $n$ & $\begin{array}{c}K_{\mathrm{C}}^{\prime}, \mathrm{M \Pi a} \sqrt{\mathrm{M}}_{\mathrm{M}} \\
K_{\mathrm{C}}^{\prime}, \mathrm{MPa} \sqrt{\mathrm{m}}\end{array}$ \\
\hline Т6 & 400 & 25 & $4.00 \cdot 10^{-2}$ & 2.4 & 24.55 \\
\hline После РКУП & 486 & 28 & $3.75 \cdot 10^{-2}$ & 2.5 & 29.06 \\
\hline
\end{tabular}

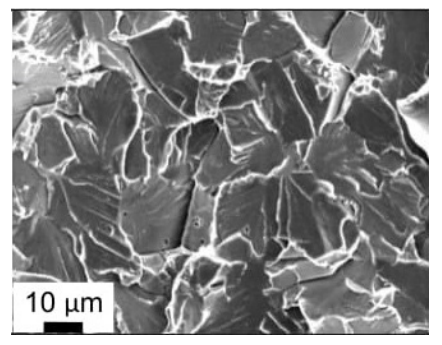

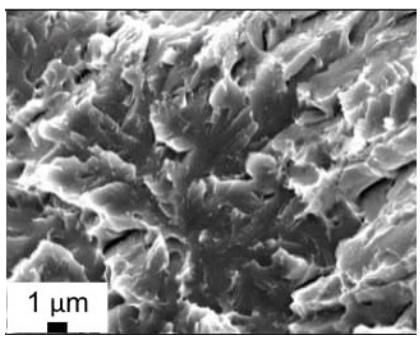

b

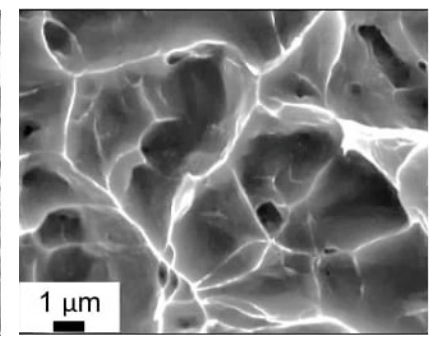

C

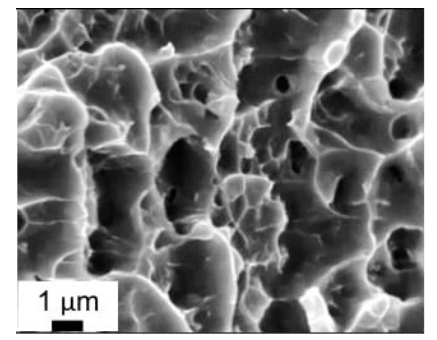

d

Рис. 3. Микрорельеф статических изломов в условиях ПД стали $09 \mathrm{MnSi5}$ (a, b) и титанового сплава Ti-6Al-4Mo (c, d) в К3 (a, c) и УМЗ (b, d) состояниях.

Fig. 3. Microrelief of static fractures under PD conditions steel $09 \mathrm{MnSi} 5$ (a, b) and titanium alloy Ti-6Al-4Mo (c, d) in CG (a, c) and UFG (b, d) states. 
шины трещины в уравнении, с критерием локальное напряженное состояние $h_{\max } / t$, где $h_{\max }-$ глубина пластической зоны под поверхностью изломов; $t$ - толщина образца или детали.

Показана принципиальная возможность оценки статической трещиностойкости $\left(K_{1 \mathrm{C}}\right.$ или $\left.K_{\mathrm{C}}\right)$ не только КЗ, но и УМЗ материала разрушенного образца или детали с различным типом кристаллической решетки по глубине пластической зоны под поверхностью изломов.

Благодарности / Acknowledgements. Авторы благодарят научных сотрудников НИИ ПТ ТГУ М.Л. Линдерова, Е. Д. Мерсона и научных сотрудников НИИ ФПМ УГАТУ О.Б. Кулясову, М.А. Никитину, А. В. Ганеева за помощь в проведении экспериментальной части работы. Работа выполнена при бинансовой поддержке РФФИ (проект 18-08-00340_a). / The authors are grateful to M. L. Linderov, E.D. Merson and scientific staffs of the Research Institute of PAM USATU O.B. Kulyasova, M.A. Nikitin, A. V. Ganeev for help in carrying out the experimental part of the work. This work was supported by the Russian Fond Fundamental Investigation (RFFI) [grant number 18-08-00340_a].

\section{Литература/References}

1. M. Srinivas, G. Malakondaiah, R. W. Armstrong, P. Rama Rao. Acta Metall. Mater. 39 (5), 807 (1991). $\underline{\text { Crossref }}$

2. I. Sabirov, R.Z. Valiev, I.P. Semenova, R. Pippan. Metallurgical and materials transactions A. 41, 727 (2010). Crossref

3. C.F. Shih. J. Mech. Phys. Solids. 29 (4), 305 (1981). Crossref

4. M. Srinivas, G. Malakondaiah, P. RamaRao. Acta Metall. Mater. 41, 1301 (1993). Crossref

5. R. Boyer, G. Welsch, E. W. Collings. Materials Properties Handbook: Titanium Alloys. USA, ASM Internationa (1998) $1048 \mathrm{p}$.

6. G. V. Klevtsov, E. V. Bobruk, I.P. Semenova, N. A. Klevtsova, R.Z. Valiev. Strength and fracture mechanisms of bulk nanostructured metallic materials. Ufa, UGATU (2016) 240 p. (in Russian) [Г. В. Клевцов, Е. В. Бобрук, И.П. Семенова, Н.А. Клевцова, Р.З. Валиев. Прочность и механизмы разрушения объемных наноструктурированных металлических материалов. Уфа, УГАТУ (2016) 240 с.]

7. R.Z. Valiev, A.P. Zhilyaev, T.G. Langdon. Bulk Nanostructured Materials: Fundamentals and Applications. USA, TMS, WILEY (2014) 440 p. Crossref

8. A.J. McEvily. Metal Failures: Mechanisms, Analysis, Prevention. New York, Wiley \& Sons (2002) 324 p.

9. G. V. Klevtsov, L. R. Botvina, N. A. Klevtsova, L. V. Limar. Fractodiagnosis of the fracture of metallic materials and structures. Moscow. MISiS (2007) 264 p. (in
Russian) [Г.В.Клевцов, Л. Р. Ботвина, Н. А. Клевцова, Л.В. Лимарь. Фрактодиагностика разрушения металлических материалов и конструкций. Москва, МИСиС (2007) 264 с.]

10. F. Wetscher, R. Stock, R. Pippan. Fracture Processes in Severe Plastic Deformed Rail Steels. Proceedings of the 16th European Conference on Fracture. Alexandropoulos, Greece (2006) p.1.

11. D. Taylor. Engineering Fracture Mechanics. 75, 1696 (2008). Crossref

12. K. Hellan. Introduction to Fracture Mechanics. Moscow, Mir (1988) 364 p. (in Russian) [К. Хеллан. Введение в механику разрушения. Москва, Мир (1988) 364 с.]

13. L. C. Moroz. Mechanics and physics of deformation and fracture. Leningrad, Mashinostroyeniye (1984) 224 p. (in Russian) [Л. С. Мороз. Механика и физика деформаций и разрушения. Ленинград, Машиностроение (1984) 224 c.]

14. G. V. Klevtsov, L. R. Botvina, N.A. Klevtsova, A. P. Fot. Metal Science and Heat Treatment. 52 (7), 396 (2010). Crossref

15. G. V. Klevtsov, R. Z. Valiev, N. A. Klevtsova, I. P. Semenova, I. N. Pigaleva, M.L. Linderov. Letters on Materials. 10 (1), 16 (2020). (in Russian) [Г. В. Клевцов, Р.3. Валиев, Н.А. Клевцова, И.П. Семенова, И.Н. Пигалева, М.Л. Линдеров. Письма о материалах. 10 (1), 16 (2020).] Crossref

16. R 50-54-52/2-94. Calculations and strength tests. Method of X-ray structural analysis of fractures. Determination of the fracture characteristics of metallic materials by the X-ray method. Moscow, VNIINMASH Gosstandart of Russia (1994) 28 p. (in Russian) [Р 50-54-52/2-94. Расчеты и испытания на прочность. Метод рентгеноструктурного анализа изломов. Определение характеристик разрушения металлических материалов рентгеновским методом. Москва, ВНИИНМАШ Госстандарта России (1994) 28 c.]

17. A. Hohenwarter, R. Pippan. Acta Materialia. 61, 2973 (2013). Crossref

18. A. Hohenwarter, R. Pippan. Fracture toughness and fatigue crack propagation measurements in ultrafine grained iron and nickel. USA, TMS (2008) 183 p.

19. L. R. Botvina, M.I. Alymov, M. R. Tutin, T.B. Petersen, S. A. Tihomirov, N. A. Solovev. Russian Nanotechnologies. 2 (1-2), 106 (2007). (in Russian) [Л.Р. Ботвина, М.И. Алымов, М.Р. Тютин, Т.Б. Петерсен, С.А. Тихомиров, Н.А. Соловьев. Российские нанотехнологии. 2 (1-2), 106 (2007).]

20. G. V. Klevtsov. Factory laboratory. 57 (3), 32 (1991). (in Russian) [Г. В. Клевцов. Заводская лаборатория, 57 (3), 32 (1991).]

21. T. Hanamura, F. Yin, K. Nagai. ISIJ Int. 44, 610 (2004). $\underline{\text { Crossref }}$ 